\title{
Study on the Bidirectional Regulation of Skin Regeneration by Tension
}

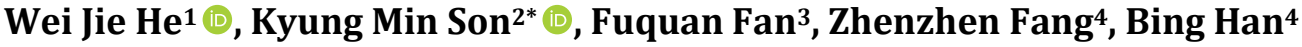 \\ ${ }^{1}$ Department of Plastic and Reconstructive Surgery, Graduate School of Medicine, Chosun University, Gwangju, Korea \\ ${ }^{2}$ Department of Plastic and Reconstructive Surgery, College of Medicine, Chosun University, Gwangju, Korea \\ ${ }^{3}$ Qingyang Yimei Plastic Surgery Hospital, Guiyang, China \\ ${ }^{4}$ Jinshui Meike Medical Beauty Clinic, Zhengzhou, China \\ Email: *8love17@hanmail.net
}

How to cite this paper: He, W.J., Son, K.M., Fan, F.Q., Fang, Z.Z. and Han, B. (2021) Study on the Bidirectional Regulation of Skin Regeneration by Tension. Modern Plastic Surgery, 11, 14-21. https://doi.org/10.4236/mps.2021.111003

Received: October 21, 2020

Accepted: January 10, 2021

Published: January 13, 2021

Copyright (C) 2021 by author(s) and Scientific Research Publishing Inc. This work is licensed under the Creative Commons Attribution International License (CC BY 4.0).

http://creativecommons.org/licenses/by/4.0/

(cc) Open Access

\begin{abstract}
Objective: To explore the related factors of tension on wound skin healing and its solution. Methods: According to the analysis and discussion of 60 trauma patients admitted to the emergency department of our hospital, they were randomly divided into two groups, 30 patients in each group (Observation and control group). The other group was systematically studied for the related factors affecting wound healing and we gave the relevant nursing measures to the control group. Results: The healing rate of the two groups were compared after treatment and nursing. The observation group was better than the control group, and the difference was statistically significant $(\mathrm{P}<$ 0.001). Conclusion: Effective reduction of wound tension can induce immune response and have obvious effect on skin repair and regeneration. On the other hand, the prevention and treatment of abnormal hyperplasia and scar were also improved. Avoid other factors affecting wound healing, strengthen postoperative management, reduce scar formation and promote skin regeneration.
\end{abstract}

\section{Keywords}

Wound Repair, Tension, Bidirectional Regulation, Organizational Restructuring

\section{Introduction}

After skin injury, the healing result in regeneration, normal scar tissue repair, hypertrophic scar tissue repair or scar Keloid formation. The role of chemical factors in wound healing has been extensively studied, and although there is an evidence of mechanical forces, the effects are far from well defined. The latest 
progress of the role of mechanical force in skin wound healing was reviewed. Comparison of different species can provide an understanding of the regulators. Interestingly, some findings suggest that tension can induce an immune response, which provides a link between mechanical and chemical forces. Structure and function have been shown to be regulated [1] both chemically and mechanically. The increase of skin tension can lead to abnormal hyperplasia of scar. Although the regeneration ability of human skin after injury is limited, the bidirectional regulation of tension will obviously improve it, thus restoring the whole structure and function of organs.

\section{Skin Mechanics}

The skin is the largest organ of the human body. It is mechanically very complex tissue. The cuticle $(10-15 \mu \mathrm{m})$, the living epidermis $(100-150 \mu \mathrm{m})$, the dermis (subdivided into papillary and reticular dermis $\approx 2 \mathrm{~mm}$ ) and subcutaneous are present below the skin surface [2]. Although the cuticle is part of the epidermis, it is generally considered as a separate layer due to its specific barrier properties. It is composed of inactive cells, very strong, but flexible and wrinkled. The epidermis is mainly composed of cells that migrate to the skin surface. When cells are closer to the cuticle, they become more keratinized. With age, the ups and downs become smaller and smaller. The dermis is mainly composed of very dense collagen and elastin fiber networks, which dominate the mechanical behavior of the whole skin. The deepest skin layer, subcutaneous or subcutaneous adipose tissue, consists of loose adipose connective tissue. The dermis contains microstructures such as blood vessels, lymphatic vessels, nerve endings, sweat glands, hair follicles and different cell types [3]. People often ignore the influence of different layers on mechanical properties.

\subsection{Tension and Immune Response}

Tissue contraction is part of normal wound healing. Tension arises during cell-mediated tissue contraction. Fibroblasts (FB) are contractile and because the tissue itself has a certain tension (ability to resist deformation), the FB is subjected to resistance from the surrounding contracting? The force depends on the deformation ability of the extracellular matrix, also known as tissue compliance. The worse the deformation ability of extracellular matrix, the worse the tissue compliance and the greater the corresponding resistance. However, the resistance determines the intracellular tension [4], and mechanical stimulation plays an important role in triggering the interaction between cells and extracellular matrix and producing signals that regulate the phosphorylation of cytokine receptors and cell proliferation. As long as there is mechanical tension in the tissue, cell proliferation and biosynthesis will continue; once tension is alleviated, even if growth factors persist, cells will be transformed into non-proliferative types and begin to degenerate. This result indicates that increased skin wound tension leads to increased scar formation, and can also explain the role of ex- 
ogenous pressure in reducing scar contracture. The results showed that the transplantation of full-thickness skin or blade thick skin could affect granulation tissue proliferation to varying degrees and accelerate the rapid completion [5] of FB life cycle. Whether this phenomenon is related to skin graft (especially full thick skin graft) can promote tension relief to some extent.

\subsection{Tension and Scar}

Scar formation is related to the degree of injury, local inflammation, immune response, skin tension and many other factors. The tension factor is closely related to the degree of scar after healing. It is still a challenging task to reduce the tension and make the incision not only obtain rapid and good healing and not obvious postoperative scar. In recent years, the main purpose of the improved suture technique is to observe the treatment and prevention of pathological scar, that is, hypertrophic scar and keloid, while the degree of tension reduction of subcutaneous suture technique is less. It also involves the evaluation of physiological scar degree and related comparative studies. In recent years, the extension-reducing suture of distal subcutaneous transverse mattress is a kind of peak and method, which can make the tension-reducing incision better. Whether under static tension or periodic dynamic tension, the distal subcutaneous transverse mattress tension-reducing suture is subcutaneous buried vertical mattress suture. Both can effectively maintain the tension-free state of the posterior edge and the adjacent skin on both sides, this suture method can be used in facial scar shaping, which can effectively reduce the tension of the knife-edge, make the cut edge good valgus and alignment, and greatly reduce the dermis suture. Compared with the traditional suture, scar width and POSAS scar scale (Patient and Observer Scar Assessment Scale) score showed that the scar degree was lighter, and itching, pain and other discomfort symptoms were lighter. The degree of scar appearance in the observation group and the control group was observed one week later (Figure 1).

Skin tension is determined by the following factors: 1) the degree of skin tissue defect; 2) the inherent tension of skin tissue and the size of local skin tension are related to skin tension relaxation line [6]. Animal experiments confirmed that with the increase of tension, the width and tension intensity of scar also increased. A relationship between wound tension and scar width confirms to a nonlinear equation [7]. For different parts of the body, skin tension is different. The site of high skin tension often becomes the prone part of pathological scar. Such as keloid often appears in the anterior chest and deltoid muscle and other areas of high skin tension. Studies have shown that FB under tension produces more collagen, and the collagen fibers [8]. And through " $Z$ " shape surgery as far as possible to repair the defective tissue or skin transplantation to alleviate skin tension is one kind.

Treatment: One of the factors of low incidence of hypertrophic scar in the elderly is skin relaxation, low tension and low collagen fiber response. 


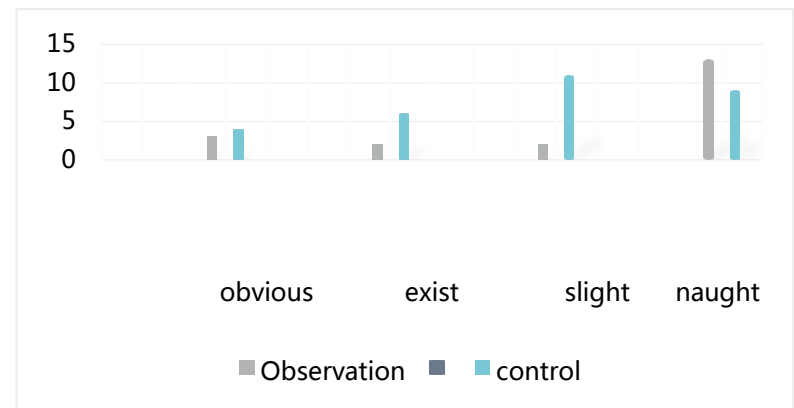

Figure 1. Degree of scar.

Early studies have found that local blood flow disorders caused by pressure can stabilize mast cells, cause tissue hypoxia, and reduce a2_macroglobulin in local plasma that inhibits collagenase activity. Thus changing collagen metabolism rate, collagen synthesis is reduced, degradation is accelerated, and myofibroblasts (Myofibroblasts, MFB) are degraded, and lysosomal enzymes that can degrade proteoglycan are released, which is beneficial to collagen remodeling [9]. This may be one of the mechanisms by which stress therapy reduces scar formation. To assess postoperative scar formation, an improved version of the modified Vancouver Scar Scale (mVSS) was used, focusing on the size of the scar region (Table 1).

The medical records were evaluated for preoperative and postoperative photographs taken at least one week after surgery. The nonparametric Wilcoxon symbolic rank test was used to analyze the difference of (mVSS) scores before and after operation. All statistical analyses were performed using the SPSS 23.0 version (IBM Corp., Armonk, NY, USA) of the data. Statistical significance is defined as the $\mathrm{P}<0.05$.

At least a week after surgery, the aesthetic satisfaction of patients was evaluated orally by dealing with the evaluation of wound scar sites (Table 2). Presenting the following four questions, full score close to five indicates the higher satisfaction: (Q1) are you satisfied with the postoperative scar at the wound site? (1-5); (Q2) are you satisfied with the postoperative contour of the wound site? (1-5); (Q3) are you satisfied with the wound site after operation? (1-5); And (Q4) are you satisfied with the overall results of the operation? (1-5) Of the $60 \mathrm{pa}-$ tients, 18 patients had a slight scar tendency, 23.33 percent. It is certain that the patient is generally satisfied.

\section{Effects of Tension and Blood Vessels on Skin Regeneration}

As a normal biological process, wound healing is achieved through four precise and highly programmed stages: hemostasis, inflammation, proliferation and remodeling. For successful wound healing, all four stages must be in the correct order and time frame [10], among which the importance of tension can be seen. Many factors interfere with one or more stages of the process, leading to impro- 
per or impaired wound healing. This review reviews recent literature on the most important factors affecting skin wound healing as well as underlying cellular and/or molecular mechanisms. Factors discussed include oxygenation, infection, age, sex hormones, tension, diabetes, obesity, drugs, alcoholism, smoking and nutrition.

Table 1. Modified vancouver scar scale.

\begin{tabular}{|c|c|}
\hline Scar characteristics & Score \\
\hline \multicolumn{2}{|l|}{ Vascularity } \\
\hline Normal & 0 \\
\hline Pink & 1 \\
\hline Red & 1 \\
\hline Purple & 1 \\
\hline \multicolumn{2}{|l|}{ Pigmentation } \\
\hline Normal & 0 \\
\hline Hypopigmentation & 1 \\
\hline Hyperpigmentation & 1 \\
\hline \multicolumn{2}{|l|}{ Pliability } \\
\hline Normal & 0 \\
\hline Supple & 1 \\
\hline Yielding & 1 \\
\hline Firm & 0 \\
\hline Ropes & 1 \\
\hline Contracture & 1 \\
\hline \multicolumn{2}{|l|}{ Height (mm) } \\
\hline \multirow[t]{2}{*}{ Flat } & 0 \\
\hline & 1 \\
\hline \multirow[t]{2}{*}{$2-5$} & 2 \\
\hline & 0 \\
\hline \multicolumn{2}{|l|}{ Depression $\left(\mathrm{cm}^{2}\right)$} \\
\hline \multirow[t]{2}{*}{ Flat } & 0 \\
\hline & 0 \\
\hline \multirow[t]{2}{*}{$4-9$} & 1 \\
\hline & 1 \\
\hline Total score & 14 \\
\hline
\end{tabular}


Table 2. Postoperative aesthetic satisfaction scores.

\begin{tabular}{cccccc}
\hline \multirow{2}{*}{ Patient No. } & \multicolumn{4}{c}{ Satisfaction score } & \multirow{2}{*}{ Total } \\
\cline { 2 - 4 } & Q1 & Q2 & Q3 & Q4 & \\
\hline 1 & 5 & 4 & 4 & 4 & 17 \\
2 & 5 & 4 & 4 & 5 & 18 \\
3 & 3 & 5 & 4 & 3 & 15 \\
4 & 4 & 5 & 4 & 5 & 18 \\
5 & 5 & 4 & 5 & 5 & 19 \\
6 & 4 & 3 & 4 & 3 & 14 \\
7 & 4 & 5 & 3 & 4 & 16 \\
8 & 5 & 5 & 4 & 4 & 17 \\
Mean \pm SD & $3.87 \pm 0.35$ & $4.37 \pm 0.35$ & $4.00 \pm 0.03$ & $4.12 \pm 0.45$ & $16.75 \pm 1.47$ \\
\hline
\end{tabular}

Higher scores (5 points) indicate greater satisfaction. Q1: Are you satisfied with the postoperative scar at the deformity site? Q2: Are you satisfied with the postoperative contour of the deformity site? Q3: Are you satisfied with the wound site after operation? Q4: Are you satisfied with the overall results of surgery?

Leighton [11] capillary blood flow in dilated flap repair is mainly related to hypoxia and mechanical tension after dilatation. Recent studies have shown that external tension acts on skin tissue and increases the differentiation of skin tissue cells and the proliferation of vascular networks. Mechanical forces affect the development of physiological and pathological tissues and the formation of new blood vessels. At present, many studies have actively explored to know the application of mechanical biological processes to control angiogenesis in living tissues [12]. Under the action of tension, the skin blood vessels will have some morphological changes. The tension produced by tissue dilatation causes sympathetic nerve rupture, that is, desympathetic innervation, which leads to the repositioning and dilatation of hypoxic blood vessels in the tissue. Traffic branches open, capillary alternate opening increased, blood flow speed increased, similar to flap delay phenomenon. Therefore, it can be considered that tissue dilatation is a delayed form of flap, a repeated mechanical dilatation process for the dilated flap, and a continuous hypoxia training process for the flap. Animal experiments have confirmed that the microvascular diameter of the dilated skin increases periodically, and the increase of microvascular density increases linearly in a certain period of time. The ischemia caused by tissue dilatation induces the angiogenesis of the flap (Figure 2).

Vascular reconstruction caused by tension is a complex process, including the synergistic expression of angiogenic factors and receptors, as well as the regulation of oxygen in tissues and the direct effect of mechanical pull on living tissues. Discussion of flap vascular changes caused by dilator dilation [13], Besides observing the vascular diameter and its distribution density, it is mainly the effect of tissue ischemia and hypoxia caused by mechanical tension on vascular changes, and the observation of these changes requires the observation of relevant factors, cell expression and changes through specific detection methods to 

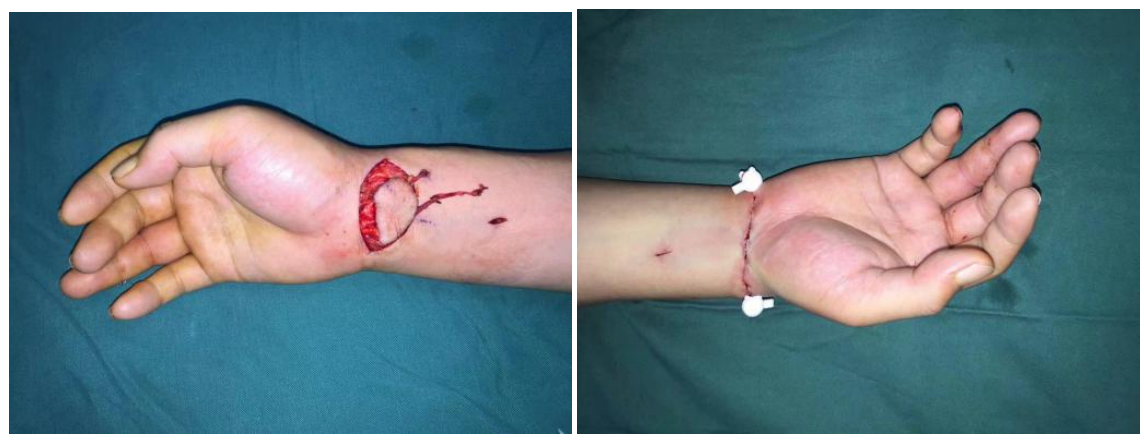

Figure 2. Tension-free suture.

indirectly reflect the changes of blood vessels. At present, the relevant factors are involved in the regulation mechanism of the vascular change process of dilated flap [14], and the results and mechanisms of the interaction need to be further studied. Therefore, we believe that the main direction of this field is to extract more specific angiogenic factors in dilated flaps, which provides a new basis for the mechanism of further dilated flap angiogenesis.

\section{Conclusion}

Our experience is that the wound tension can affect wound healing. Therefore, in order to ensure good healing of suture wound, it is necessary to minimize suture tension. Previous studies have shown that skin tissue tension around the wound has a significant effect on wound healing. The microtubules and microfilaments of tissue repair cells tend to the direction of local tissue tension line, which affects the direction of wound contraction and cell migration. Reducing tension can not only promote the growth of immune cells, but also promote the regeneration of blood vessels, tissue regeneration accelerates and can also reduce scar formation. Hyperplastic scar is pathological scar caused by excessive synthesis and deposition of collagen [15]. Skin mechanical tension can cause fibroblast proliferation and transformation through various mechanisms, regulate protein excretion, inhibit apoptosis, induce pathological scar formation, and promote its proliferation and expansion. Tension-related therapy, surgical incision design, suture, and skin tensioner, wound tape, pressure treatment can effectively inhibit scar hyperplasia and avoid the occurrence and recurrence of pathological scars and promote skin regeneration.

\section{Conflicts of Interest}

The authors declare no conflicts of interest regarding the publication of this paper.

\section{References}

[1] Agha, R., Ogawa, R., Pietramaggiori, G. and Orgill, D.P. (2011) A Review of the Role of Mechanical Forces in Cutaneous Wound Healing. Journal of Surgical Research, 171, 700-708. https://doi.org/10.1016/j.jss.2011.07.007 
[2] Oomens, C.W., van Campen, D.H. and Grootenboer, H.J. (1987) In Vitro Compression of a Soft Tissue Layer on a Rigid Foundation. Journal of Biomechanics, 20, 923-935. https://doi.org/10.1016/0021-9290(87)90321-6

[3] Ali, S.M., Bonnier, F., Tfayli, A., Lambkin, H.A., Flynn, K., McDonagh, V., Healy, C., Lee, T.C., Lyng, F.M. and Byrne, H.J. (2012) Raman Spectroscopic Analysis of Human Skin Tissue Sections Ex-Vivo: Evaluation of the Effects of Tissue Processing and Dewaxing. Journal of Biomedical Optics, 18, Article ID: 061202.

https://doi.org/10.1117/1.JBO.18.6.061202

[4] Arora, P.D., Narani, N. and McCulloch, C.A. (1999) The Compliance of Collagen Gels Regulates Transforming Growth Factor-Beta Induction of Alpha-Smooth Muscle Actin in Fibroblasts. The American Journal of Pathology, 154, 871-882. https://doi.org/10.1016/S0002-9440(10)65334-5

[5] Rudolph, R. (1979) Inhibition of Myofibroblasts by Skin Grafts. Plastic and Reconstructive Surgery, 63, 473-480. https://doi.org/10.1097/00006534-197904000-00005

[6] Wang, Z.G. (1998) Wound Healing and Tissue Repair. Shandong Science and Technology Press, Jinan, 264.

[7] Burgess, L.P.A., Morin, G.V., Rand, M., Vossoughi, J. and Hollinger, J.O. (1990) Wound Healing: Relationship of Wound Closing Tension to Scar Width in Rats. Archives of Otolaryngology-Head \& Neck Surgery, 116, 798-802. https://doi.org/10.1001/archotol.1990.01870070046008

[8] Rockwell, W.B., Cohen, I.K. and Ehrlich, H.P. (1989) Keloids and Hypertrophic Scars View. Plastic and Reconstructive Surgery, 84, 827-837. https://doi.org/10.1097/00006534-198911000-00021

[9] Choi, M.H., He, W.J., Son, K.M., Choi, W.Y. and Cheon, J.S. (2020) The Efficacy of Dermofat Grafts from the Groin for Correction of Acquired Facial Deformities. Archives of Craniofacial Surgery, 21, 92-98. https://doi.org/10.7181/acfs.2020.00038

[10] Dreifke, M.B., Jayasuriya, A.A. and Jayasuriya, A.C. (2015) Current Wound Healing Procedures and Potential Care. Materials Science \& Engineering C: Materials for Biological Applications, 48, 651-662. https://doi.org/10.1016/j.msec.2014.12.068

[11] Leighton, W.D., Russell, R.C., Marcus, D.E., Eriksson, E., Suchy, H. and Zook, E.G. (1988) Experimental Pretransfer Expansion of Free-Flap Donor Sites. Plastic and Reconstructive Surgery, 82, 69-75. https://doi.org/10.1097/00006534-198807000-00013

[12] Kilarski, W.W., Samolov, B., Petersson, L., Kvanta, A. and Gerwins, P. (2009) Biomechanical Regulation of Blood Vessel Growth during Tissue Vascularization. Nature Medicine, 15, 657-664. https://doi.org/10.1038/nm.1985

[13] Wang, X.Y., Li, C.C., Zheng, Y., et al. (2011) Bone Marrow-Derived Stem Cells Contribute Skin Regeneration in Skin and Soft Tissue Expansion. Journal of Cellular Physiology, 226, 2834-2840. https://doi.org/10.1002/jcp.22634

[14] Johnson, K.E. and Wilgus, T.A. (2014) Vascular Endothelial Growth Factor and Angiogenesis in the Regulation of Cutaneous Wound Repair. Advances in Wound Care, 3, 647-661. https://doi.org/10.1089/wound.2013.0517

[15] Ma, D., Chen, L., Shi, J., et al. (2020) Pigment Epithelium-Derived Factor Attenuates Angiogenesis and Collagen Deposition in Hypertrophic Scars. Wound Repair and Regeneration, 28, 684-695. https://doi.org/10.1111/wrr.12828 